\title{
Depressão e ansiedade entre estudantes da área de saúde
}

\author{
Depression and anxiety among medical students
}

\section{Tania Correa de Toledo Ferraz Alves}

Alves TCTF. Depressão e ansiedade entre estudantes da área de saúde / Depression and anxiety among medical students. Rev Med (São Paulo). 2014 jul.-set.;93(3):101-5.

RESUMO: Depressão e ansiedade são sintomas frequentemente observados entre estudantes da área da saúde, mais especificamente medicina. A presença destes sintomas, bem como a não identificação precoce dos mesmos pode levar a um impacto muito grande no desenvolver da carreira médica do aluno, com queda de rendimento, aumento do risco de abandono/desistência do curso e aumento do risco de suicídio. Esta revisão visa abordar esses aspectos e discutir possíveis abordagens.

DESCRITOES: Depressão/psicologia; Ansiedade/psicologia; Suicídio/psicologia; Medicina; Educação de graduação em medicina; Estudantes de medicina/psicologia; Literatura de revisão como assunto.

\begin{abstract}
Depression and anxiety symptoms are often observed among students in the health care area, particularly in medical course. The presence of these symptoms can lead to an impact on the development of the medical student's career, falling to perform in the course, increased risk of abandonment / discontinuance of the course and increased risk of suicide. This review aims to address these issues and discuss possible approaches.
\end{abstract}

KEY WORDS: Depression/psychology; Anxiety/psychology; Suicide/psychology; Medicine; Education, medical, undergraduate; Students, medical/psychology; Review literature as topic.

\footnotetext{
Médica Supervisora do Instituto de Psiquiatria do Hospital das Clínicas da Faculdade de Medicina da Universidade de São Paulo IPq-HCFMUSP; Professora Colaboradora Médica do Departamento de Psiquiatria da Faculdade de Medicina da Universidade de São Paulo - FMUSP.

Endereço para correspondência: Tania C. T. F. Alves. Instituto de Psiquiatria do HCFMUSP. R.: Dr. Ovídio de Campos, s/n, $3^{\circ}$ andar, sala 14 Ceapesq. São Paulo, SP, Brasil. E-mail: tania.ctfalves@hc.fm.usp.br.
} 


\section{INTRODUÇÃO}

$\mathbf{E}$ studantes da área de saúde são propensos a apresentar sintomas depressivos e ansiosos durante seu curso, que podem impactar o seu futuro profissional. Diversos estudos da literatura apontam o risco maior entre os estudantes do curso médico ${ }^{1-4}$ quando comparado a outros cursos de diferentes áreas. Nos cursos médicos, o internato de medicina é o período no qual o estudante tem a oportunidade de vivenciar de forma mais realista e intensa as experiências da prática médica e assumir uma nova postura diante dos pacientes. Ele deixa de ser apenas um mero observador, sustentado em um conhecimento principalmente teórico, para ser ativo, intervir, opinar em condutas e exercitar em sua plenitude o contato médico-paciente, mesmo que sob orientação e tutoria de seus preceptores ${ }^{5}$. A presença de sintomas depressivos e ansiosos relevantes leva ao estudante dificuldades em seguir o curso, aumenta o número de abandonos e aumenta o risco de desenvolvimento de quadros de dependência química e até suicídio ${ }^{1}$. Esta revisão foca neste tema relevante e importante para o planejamento de estratégias que possam minimizar ou diagnosticar precocemente aqueles alunos em risco.

\section{SINTOMAS ANSIOSOS E ESTUDANTES DE MEDICINA}

A ansiedade é entendida como uma emoção normal da vivência humana. Entretanto sua conceituação ainda é difícil, bem como a delimitação da ansiedade normal e da patológica. Costuma ser percebida como um estado de alerta, que leva à tensão e ao gasto energético, associado a uma percepção do ambiente aumentada voltada a um perigo percebido conscientemente ou não. A ansiedade geralmente leva a uma mudança do estado físico e psíquico, com projeção de futuro muitas vezes negativa, porém, com possibilidade de modificação do curso de ação. Por exemplo, o exame de residência médica ao final do curso médico em geral determina aos alunos do sexto ano um estado de tensão física e psíquica, com pensamentos de ansiedade antecipatória "não irei passar", "é muito concorrido". Quando a ansiedade está no campo normal, esta situação gera modificação do comportamento ambiental ("luta"), na qual o estudante foca e se disciplina, deixa de sair muitas vezes, e aumenta seu rendimento e sua produtividade. Com isso não se confirma o pressuposto inicial, de forma que "por medo de não passar, aumentou seu rendimento e atingiu seu resultado". Por outro lado, a ansiedade patológica pode levar a uma resposta de congelamento ("fuga"), na qual se adota comportamentos de procrastinação, baixa de rendimento e aumento das situações de saída com amigos ("para desestressar"). A esse comportamento o resultado final em geral e a confirmação do pressuposto inicial de falha e incompetência, de forma que o estudante perde a auto-estima, entrando num ciclo vicioso.

É interessante a fala de Epicteto (55-135 d.C.), que foi um dos grandes mestres do Estoicismo e dedicou sua vida a responder a duas questões fundamentais: "Como viver uma vida plena e feliz?" e "Como ser uma pessoa com boas qualidades morais?".

\begin{abstract}
"De todas as coisas existentes algumas estão sob o nosso poder e outras não. Debaixo do nosso poder estão o pensamento, o impulso, a vontade de adquirir e a vontade de evitar e, numa palavra, tudo que resulta das nossas ações. "

"O que perturba a mente dos homens não são os eventos, mas os seus julgamentos sobre os eventos".

"Não questione se os eventos deveriam acontecer tal como você desejaria, mas deixe ser sua vontade que os eventos devam acontecer tal como eles ocorrem, e você terá paz".
\end{abstract}

Epicteto acreditava que a meta principal da filosofia é ajudar as pessoas comuns a enfrentar positivamente os desafios cotidianos e a lidar com as inevitáveis perdas, decepções e mágoas da vida. A raiz de toda a frustração e infelicidade reside, segundo Epicteto, na interpretação fantasiosa da realidade e nos juízos falsos que fazemos sobre ela. Assim a separação entre ansiedade normal e patológica, pode ser dada menos pela intensidade dos sintomas, mas sim principalmente pela interpretação desses sintomas frente ao ambiente pelo sujeito ${ }^{6}$. A mesma intensidade de sintomas pode, para um indivíduo levar a uma resposta de aumento de produtividade e ser propulsora e em outro sujeito levar a paralisia e piora da performance ${ }^{7,8}$.

Estudantes de medicina apresentam diversos aspectos do curso médico que podem levar a um aumento da ansiedade por serem fatores potencialmente estressantes, entre eles temos o contato interpessoal, vivência próxima com a morte/doença que leva a um ataque ao nosso narcisismo, elevado número de horas, em especial no internato, muitas vezes com noites insones trabalhando ${ }^{7}$. Diversos estudos apontam um aumento do nível de ansiedade quando comparam o início do curso básico e o internato $^{5,7,9-11}$. Quando se levantam estudos sobre ansiedade e curso médico, há um consenso de que estudantes de medicina são susceptíveis a maios estresse e sintomas ansiosos que os demais ${ }^{12,13}$.

Esse aumento do nível de estresse, levando em conta os conhecimentos atuais de mecanismos de adaptação, podem levar estudantes de medicina a agirem com estratégias, muitas vezes inadequadas, para compensação (mecanismos de coping stress) através da fantasia (com queda do rendimento), busca de alivio (uso de substância) ou fuga ${ }^{14-16}$. A escola médica deve estar preparada no auxílio do estudante no gerenciamento do estresse. Abordagens cognitivo-comportamentais e grupos terapêuticos podem ser úteis na redução do impacto do estresse do curso médico ${ }^{4,17,18}$. Os benefícios percebidos pelos estudantes 
submetidos a programas de gerenciamento de estresse são vários, entre eles: aumento da produtividade e do foco, melhora da capacidade de empatia, redução dos sintomas de ansiedade e depressivos, e maior leque de habilidades e competências para lidar com eventos estressantes no futuro $^{19,20}$.

\section{SINTOMAS DEPRESSIVOS E ESTUDANTES DE MEDICINA}

Diversos estudos apontam para a presença e de maior prevalência e gravidade de sintomas depressivos em estudantes do curso médico ${ }^{2,3,18,21,22}$. Quando divididos por ciclos, os estudos apontam que o ciclo pré-clínico e o internato como momentos de maior risco ${ }^{1,2,23}$. Mas a questão do porquê os estudantes de medicina têm maior risco ainda não foi completamente esclarecida. Devemos levar em conta que a Depressão é um quadro multifatorial, que inclui desde fatores genéticos como ambientais em sua fisiopatologia. Em alguns casos, os aspectos genéticos parecem ser predominantes (presença de diversos membros afetados na família), em outros aspectos ambientais parecem ter um papel relevante, bem como capacidade de gerenciamento destes fatores. Numa revisão sistemática da literatura, Dyrbye et al. ${ }^{24}$ observaram que o curso médico é um período de grande estresse psicológico para os estudantes. No primeiro semestre do curso, o estudante passa por diversas modificações da sua rotina diária, após a adaptação inicial, a carga de trabalho tende a aumentar com pico no internato/residência na qual se incluem plantões com carga muitas vezes superior a 60horas semanais de trabalho prático ${ }^{5,12}$. Outros fatores, como competitividade ${ }^{21}$, pressão constante ${ }^{25}$, presença de exame de seleção ao final do curso para residência, além de características próprias do curso como contato com morte, limites sociais, terminalidade, podem levar o estudante a aumento da ansiedade e dificuldade de gerenciamento da mesma, aumentando a vulnerabilidade aos quadros de humor $5,9,18,26-28$.

\section{Trote e depressão}

Castadelli-Maia et al. ${ }^{3}$, realizaram um estudo transversal feito com amostras de estudantes de medicina em dois momentos (2001 e 2006) procurando correlacionar a existência do trote como fator estressante e potencialmente desencadeante de sintomatologia depressiva nos estudantes de medicina. Em ambos os momentos quase $20 \%$ da amostra apresentava escores de gravidade de sintomas depressivos significativos. Uma análise qualitativa nos alunos do gênero masculino sugeriu que pouco envolvimento social e violência do trote foram correlacionados com sintomas depressivos. Estudos de seguimento são importantes para avaliação de estratégias que possam identificar precocemente e reduzir fatores potencialmente desencadeantes.

\section{SUICÍDIO E ESTUDANTES DE MEDICINA}

Desde a Antiguidade, o suicídio foi visto com controvérsia, representando, por um lado, o ato supremo da liberdade humana, e por outro, o atentado contra os deveres do homem diante da sociedade. Na Idade Média, assumiu caráter criminoso, com penas e punições previstas em lei. Na Idade da Razão, apologias e hostilidades à morte voluntária preencheram inúmeros tratados. No século XIX, a penalidade do ato suicida desapareceu de modo progressivo dos códigos do Velho Mundo. Na atualidade, a definição mais aceita caracteriza-o por morte causada por lesão, envenenamento ou sufocação, com evidência explícita ou implícita de que tenha sido auto-infligida e de que havia intenção de morrer. Os médicos figuram entre as profissões associadas com maior risco de suicídio, além disso o profissional se beneficia do seu conhecimento científico de forma que o risco de mortalidade aumenta ${ }^{1}$. O motivo do risco de suicídio ser maior na carreira médica pode estar associado a uma maior prevalência de quadros depressivos e ansiosos nesta população ${ }^{2}$. É possível que as pessoas deprimidas procurem a medicina como carreira (análise psicossomática), entretanto não podemos deixar de considerar o fato de que os indivíduos possam deprimir em função da profissão (análise bio-psicológica), o que traduz melhor as teorias modernas multifatoriais, por retratar a interação de ambos os aspectos no desencadeamento de um quadro.

Estudantes de medicina são mais propensos à depressão do que universitários não médicos ${ }^{1,2,23}$. Alexandrino-Silva et al. ${ }^{1}$ observaram que quando comparado a outras áreas de saúde, como enfermagem e farmácia, que também tem contato com pacientes, trabalho em turnos e plantões, os resultados sugerem que a prevalência de sintomas depressivos e ideação suicida foi semelhante. Embora não se observe diferença significativa, a presença de ideação suicida é um achado preocupante, visto que esses profissionais têm conhecimento para um suicídio efetivo. A identificação precoce destes sintomas é fundamental, de modo a se oferecer apoio e tratamento adequados e prevenir mortes por suicídio.

\section{TRATAMENTO DA DEPRESSÃO E ANSIEDADE NOS ESTUDANTES DE MEDICINA}

Para tratamento dos quadros ansiosos e depressivos entre os estudantes de medicina existem diversas barreiras, iniciando pelo estigma da doença mental ${ }^{22,28,29}$. Pensamentos de que o fato de sentir deprimido(a) ou ansioso(a) está associado a "fraqueza", "imaturidade", "falta do que fazer", "falta de ocupação", "pouca espiritualidade", entre outros, leva a uma demora no reconhecimento destes sintomas ${ }^{29}$. Além disso, mudanças no curso médico, associado a 
Alves TCTF. Depressão e ansiedade entre estudantes da área de saúde.

intervenções curriculares podem ter impacto na prevalência e gravidade destes sintomas ${ }^{30}$.

A identificação precoce e o encaminhamento para tratamento farmacológico e/ou psicoterápico têm impacto no desenvolvimento destes sintomas minimizando o risco de suicídio ${ }^{30}$. Em relação à ansiedade, estudos com terapia cognitivo comportamental com foco em gerenciamento de estresse tem se mostrado eficazes na prevenção de quadros ansiosos entre populações de risco, assim como estratégias não farmacológicas como atividade física e meditação ${ }^{31-33}$. Medicações em casos mais graves podem ser usadas, com foco na redução dos sintomas ansiosos. Dentre as medicações disponíveis, os antidepressivos têm papel importante na terapêutica na ansiedade ${ }^{34}$. Nos quadros depressivos, o uso de medicação em casos moderados e graves se associa a melhor prognóstico e menor chance de recaída e cronificação. As medicações disponíveis são antidepressivos com foco principal nos grupos de pouco efeito colateral, tais como inibidores seletivos da recaptura de serotonina e duais ${ }^{35,36}$. É interessante notar que quadros depressivos podem levar a impacto sobre a cognição, especificamente sobre a atenção e consequentemente sobre a memória, levando ao estudante um prejuízo funcional e dificuldade de aprendizado. Alves et al. ${ }^{37}$, mostraram que após o tratamento adequado, atingindo a remissão do quadro de humor observa-se uma reversão destes prejuízos. Finalmente, Castaldelli-Maia et al. ${ }^{3}$ sugerem que mudanças curriculares que levem a diminuição do estresse e de eventos vitais estressantes, e estimulem o gerenciamento do mesmo possa ter efeito benéfico sobre a prevalência e gravidade de depressão e ansiedade entre estudantes da área médica ${ }^{2,35}$.

\section{REFERÊNCIAS}

1. Alexandrino-Silva C, Pereira ML, Bustamante C, Ferraz AC, Baldassin S, Andrade AG, Alves TC. Suicidal ideation among students enrolled in healthcare training programs: a cross-sectional study. Rev Bras Psiquiatr. 2009;31(4):338-44. http://dx.doi.org/10.1590/S1516-44462009005000006.

2. Baldassin S, Silva N, de Toledo Ferraz Alves TC, CastaldelliMaia JM, Bhugra D, Nogueira-Martins MC, et al. Depression in medical students: cluster symptoms and management. J Affect Disord. 2013;150(1):110-4. doi: 10.1016/j. jad.2012.11.050.

3. Castaldelli-Maia JM, Martins SS, Bhugra D, Machado MP, Andrade AG, Alexandrino-Silva C, et al. Does ragging play a role in medical student depression - cause or effect? J Affect Disord. 2012;139(3):291-7. doi: 10.1016/j.jad.2012.02.003.

4. Ahmed I, Banu H, Al-Fageer R, Al-Suwaidi R. Cognitive emotions: depression and anxiety in medical students and staff. J Crit Care. 2009;24(3):e1-7. doi: 10.1016/j. jcrc.2009.06.003.

5. Bassols AM, Okabayashi LS, Silva AB, Carneiro BB, Feijo F, Guimaraes GC, Cortes GN, Rohde LA, Eizirik CL. First- and last-year medical students: is there a difference in the prevalence and intensity of anxiety and depressive symptoms? Rev Bras Psiquiatr. 2014;36(3):233-40. http:// dx.doi.org/10.1590/1516-4446-2013-1183.

6. Ferguson E, Semper H, Yates J, Fitzgerald JE, Skatova A, James D. The 'dark side' and 'bright side' of personality: when too much conscientiousness and too little anxiety are detrimental with respect to the acquisition of medical knowledge and skill. PLoS One. 2014;9(2):e88606. doi: 10.1371/journal.pone.0088606.

7. Thiemann P, Quince T, Benson J, Wood D, Barclay S. Death anxiety among medical students: prevalence and implications.
BMJ Support Palliat Care. 2014;4(Suppl 1):A31-2.

8. Gramstad TO, Gjestad R, Haver B. Personality traits predict job stress, depression and anxiety among junior physicians. BMC Med Educ. 2013;13:150. doi: 10.1186/1472-6920-13150.

9. Saravanan C, Wilks R. Medical students' experience of and reaction to stress: the role of depression and anxiety. ScientificWorld J. 2014;2014:737382. doi: $10.1155 / 2014 / 737382$.

10. Lindon-Morris E, Laidlaw A. Anxiety and self-awareness in video feedback. Clin Teach. 2014;11(3):174-8. doi: 10.1111/ tct. 12103 .

11. Grochowski CO, Cartmill M, Reiter J, Spaulding J, Haviland J, Valea F, Thibodeau PL, McCorison S, Halperin EC. Anxiety in first year medical students taking gross anatomy. Clin Anat. 2014;27(6):835-8. doi: 10.1002/ca.22398.

12. Yusoff MS, Abdul Rahim AF, Baba AA, Ismail SB, Mat Pa MN, Esa AR. Prevalence and associated factors of stress, anxiety and depression among prospective medical students. Asian J Psychiatr. 2013r;6(2):128-33. doi: 10.1016/j. ajp.2012.09.012.

13. Ibrahim N, Al-Kharboush D, El-Khatib L, Al-Habib A, Asali D. Prevalence and Predictors of Anxiety and Depression among Female Medical Students in King Abdulaziz University, Jeddah, Saudi Arabia. Iranian J Public Health. 2013;42(7):726-36. Available from: http://www.ncbi.nlm. nih.gov/pmc/articles/PMC3881618/

14. Van Nguyen H, Laohasiriwong W, Saengsuwan J, Thinkhamrop $B$, Wright P. The relationships between the use of self-regulated learning strategies and depression among medical students: an accelerated prospective cohort study. Psychol Health Med. 2015;20(1):59-70. doi: 10.1080/13548506.2014.894640. 
15. Tektas OY, Paulsen F, Sel S. Test anxiety among German medical students and its impact on lifestyle and substance abuse. Med Teach. 2013;35(11):969. doi: 10.3109/0142159X.2013.786813.

16. Mancevska S, Bozinovska L, Tecce J, Pluncevik-Gligoroska J, Sivevska-Smilevska E. Depression, anxiety and substance use in medical students in the Republic of Macedonia. Bratisl Lek Listy. 2008;109(12):568-72.

17. O'Carroll PJ, Fisher P. Metacognitions, worry and attentional control in predicting OSCE performance test anxiety. Med Educ. 2013;47(6):562-8. doi: 10.1111/medu.12125.

18. Karaoglu N, Seker M. Anxiety and depression in medical students related to desire for and expectations from a medical career. West Indian Med J. 2010;59(2):196-202. Available from: http://goo.gl/90F200.

19. Velayudhan A, Gayatridevi S, Bhattacharjee RR. Efficacy of behavioral intervention in reducing anxiety and depression among medical students. Ind Psychiatry J. 2010;19(1):41-6. doi: 10.4103/0972-6748.77636.

20. Zuardi AW, Prota Fdel G, Del-Ben CM. Reduction of the anxiety of medical students after curricular reform. Rev Bras Psiquiatr. 2008;30(2):136-8. http://dx.doi.org/10.1590/ S1516-44462008005000006.

21. Gupta S, Basak P. Depression and type D personality among undergraduate medical students. Indian J Psychiatry. 2013;55(3):287-9. doi: 10.4103/0019-5545.117151.

22. Cheng DR, Poon F, Nguyen TT, Woodman RJ, Parker JD. Stigma and perception of psychological distress and depression in Australian-trained medical students: results from an inter-state medical school survey. Psychiatry Res. 2013;209(3):684-90. doi: 10.1016/j.psychres.2013.03.003.

23. Alvi T, Assad F, Ramzan M, Khan FA. Depression, anxiety and their associated factors among medical students. J Coll Physicians Surg Pak - JCPSP. 2010;20(2):122-6. doi: 02.2010/ JCPSP. 122126 .

24. Dyrbye LN, Thomas MR, Shanafelt TD. Systematic review of depression, anxiety, and other indicators of psychological distress among U.S. and Canadian medical students. Acad Med. 2006;81(4):354-73. Available from: http://goo. $\mathrm{gl} / 7 \mathrm{hwFx} 8$.

25. Yeh YC, Yen CF, Lai CS, Huang CH, Liu KM, Huang IT. Correlations between academic achievement and anxiety and depression in medical students experiencing integrated curriculum reform. Kaohsiung J Med Sci. 2007;23(8):379-86. doi:10.1016/S0257-5655(07)70001-9.

26. O'Reilly E, McNeill KG, Mavor KI, Anderson K. Looking beyond personal stressors: an examination of how academic stressors contribute to depression in Australian graduate medical students. Teach Learn Med. 2014;26(1):56-63. doi: 10.1080/10401334.2013.857330.
27. Haldorsen H, Bak NH, Dissing A, Petersson B. Stress and symptoms of depression among medical students at the University of Copenhagen. Scand J Public Health. 2014;42(1):8995. doi: 10.1177/1403494813503055.

28. Downs N, Feng W, Kirby B, McGuire T, Moutier C, Norcross W, Norman M, Young I, Zisook S. Listening to Depression and Suicide Risk in Medical Students: the Healer Education Assessment and Referral (HEAR) Program. Acad Psychiatry. 2014; 38(5):547-53. doi: 10.1007/s40596-014-0115-X.

29. Korszun A, Dinos S, Ahmed K, Bhui K. Medical student attitudes about mental illness: does medical-school education reduce stigma? Acad Psychiatry. 2012;36(3):197-204. doi: 10.1176/appi.ap.10110159.

30. Slavin SJ, Schindler DL, Chibnall JT. Medical student mental health 3.0: improving student wellness through curricular changes. Acad Med. 2014;89(4):573-7. doi: 10.1097/ ACM.0000000000000166.

31. Bogels SM, Wijts P, Oort FJ, Sallaerts SJ. Psychodynamic psychotherapy versus cognitive behavior therapy for social anxiety disorder: an efficacy and partial effectiveness trial. Depress Anxiety. 2014;31(5):363-73. doi: 10.1002/da.22246.

32. Boettcher J, Astrom V, Pahlsson D, Schenstrom O, Andersson G, Carlbring P. Internet-based mindfulness treatment for anxiety disorders: a randomized controlled trial. Behav Ther. 2014;45(2):241-53. doi: 10.1016/j.beth.2013.11.003.

33. Arch JJ, Ayers CR. Which treatment worked better for whom? Moderators of group cognitive behavioral therapy versus adapted mindfulness based stress reduction for anxiety disorders. Behav Res Ther. 2013;51(8):434-42. doi: 10.1016/j. brat.2013.04.004.

34. Baldwin DS, Anderson IM, Nutt DJ, Allgulander C, Bandelow B, den Boer JA, et al. Evidence-based pharmacological treatment of anxiety disorders, post-traumatic stress disorder and obsessive-compulsive disorder: A revision of the 2005 guidelines from the British Association for Psychopharmacology. J Psychopharmacol. 2014;28(5):403-39. doi: $10.1177 / 0269881114525674$.

35. Baldassin S, Alves TC, de Andrade AG, Nogueira Martins LA. The characteristics of depressive symptoms in medical students during medical education and training: a cross-sectional study. BMC Med Educ. 2008;8:60. doi: 10.1186/1472-69208-60.

36. Brunoni AR, Bensenor IM, Alves TC. Therapeutic interventions for vascular depression: a systematic review. Rev Bras Psiquiatr. 2011;33(4):400-9. http://dx.doi. org/10.1590/S1516-44462011000400015.

37. Alves TC, Rays J, Telles RM, Junior RF, Wajngarten M, Romano BW, Watanabe C, Busatto GF. Effects of antidepressant treatment on cognitive performance in elderly subjects with heart failure and comorbid major depression: an exploratory study. Psychosomatics. 2007;48(1):22-30. 